\title{
Microbleeds as a Prognostic Factor for Acute Subdural Hematoma
}

\author{
Tadashi YAMAgUCHI, ${ }^{1}$ Hiroki TAKAI, ${ }^{1}$ Satoshi HIRAI, ${ }^{1}$ \\ Kimihiko YOKOSUKA, ${ }^{1}$ Hiroyuki TOI, ${ }^{1}$ Kazuyuki KuWAYAMA, ${ }^{1}$ \\ Shunji MATSUBARA, ${ }^{1}$ Kazuhiro HIRANO, ${ }^{1}$ and Masaaki UNO ${ }^{1}$ \\ ${ }^{1}$ Department of Neurosurgery, Kawasaki Medical School, Kurashiki, Okayama
}

\begin{abstract}
This study investigated the frequency of poor outcome at discharge of acute subdural hematoma (SDH) patients with and without microbleeds. We retrospectively examined the records of 37 patients with acute SDH who were surgically treated with hematoma removal and received magnetic resonance (MR) imaging within 2 weeks of head injury onset. MR images were used to determine the presence or absence of microbleeds and contusional hemorrhage (CH). Patient outcome was categorized as good (moderate disability or good recovery) or poor (severely disability, vegetative state, or dead) according to the Glasgow Outcome Scale at discharge. Microbleeds were found in 23 patients $(62 \%)$ and $\mathrm{CH}$ was found in 26 patients $(70 \%)$. Fifteen patients $(41 \%)$ had both microbleeds and CH. Poor outcome at discharge was more common in SDH patients with both microbleeds and CH than in SDH patients with neither microbleeds nor CH (14/15, 93\% vs. 14/22, 64\%; $p=0.04)$. Poor outcome at discharge was more common in SDH patients under 60 years of age with microbleeds $(6 / 8,75 \%)$ than patients under 60 years of age without microbleeds $(0 / 4,0 \% ; p=0.03)$. The location of the microbleed was not related to the outcome at discharge. These results suggest that the presence of microbleeds and CH on MR images may indicate poor prognosis in patients with acute SDH.
\end{abstract}

Key words: acute subdural hematoma, microbleed, contusional hemorrhage, magnetic resonance imaging

\section{Introduction}

Acute subdural hematoma (SDH) complicates approximately $20 \%$ of severe traumatic brain injuries. ${ }^{4,12,13,18)}$ The overall mortality rate in patients with acute $\mathrm{SDH}$ who require surgery is $40 \%$ to $60 \% .{ }^{4,13)}$ Prognostic indicators of acute SDH are age, Glasgow Coma Scale score, pupillary reactivity, SDH volume, and midline brain shift. ${ }^{4,6,9,14)}$ Microbleeds identified on $\mathrm{T}_{2}{ }^{*}$-weighted magnetic resonance (MR) images correlate with outcome in patients with diffuse axonal injury. ${ }^{7,16,20)}$ However, the relationship between microbleeds and prognosis in patients with acute SDH is not known. The present study investigated the relationship between microbleeds and outcome in patients with acute SDH.

Received July 12, 2012;

Accepted August 17, 2012

\section{Materials and Methods}

We retrospectively reviewed the medical records of all patients who were admitted to the University Hospital of Kawasaki Medical School between January 2005 and October 2011 with acute SDH and who were surgically treated with hematoma removal with or without decompressive craniectomy. Patients were included in this study if they underwent MR imaging within 2 weeks of the onset of head injury. Patients who underwent MR imaging more than 15 days after SDH onset, had conservative treatment, received burr hole surgery, or were diagnosed with acute SDH in the posterior cranial fossa were excluded. The institutional ethics committee of Kawasaki Medical School approved the study protocol.

MR imaging was performed using a $1.5 \mathrm{~T}$ Signa Horizon, Signa EXCITE (GE Healthcare, Chalfont St. Giles, Buckinghamshire, UK) or Excelart Vantage Atlas (Toshiba Medical Systems Corp., Otawara, Tochigi) scanner. Microbleeds were diagnosed on 

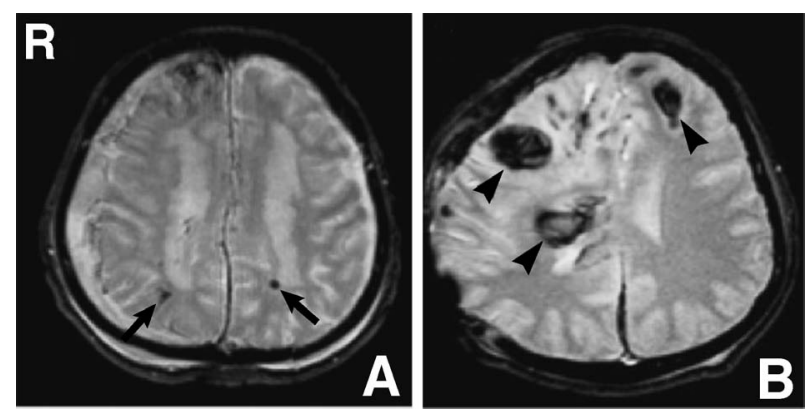

Fig. $1 \mathrm{~T}_{2}{ }^{*}$-weighted magnetic resonance images showing microbleeds (A: arrows) and contusional hemorrhage (B: arrowheads).

axial $\mathrm{T}_{2}{ }^{*}$-weighted gradient echo images obtained with a repetition time of $600-700 \mathrm{msec}$ and an echo time of $20 \mathrm{msec}$, and were defined as focal areas of very low signal intensity, smaller than $10 \mathrm{~mm}$ in size (Fig. 1A). Contusional hemorrhage (CH) was defined as areas of very low signal intensity larger than 10 $\mathrm{mm}$ in size (Fig. 1B).

Patient outcome was classified according to the Glasgow Outcome Scale at discharge. Patients with moderate disability or good recovery were defined as having a good outcome. Patients with severe disability and those in a vegetative state or dead were defined as having a poor outcome. The distribution of good and poor outcomes was compared across patients with and without microbleeds, and with and without $\mathrm{CH}$, using Fisher's exact test. A value of $p<0.05$ was considered significant.

\section{Results}

We reviewed the records of 81 patients diagnosed with acute SDH who received surgical treatment with hematoma removal. Of these, 37 patients received MR imaging within 2 weeks of head injury onset and formed the sample for the current study. Clinical and demographic characteristics of these patients are shown in Table 1 . The mean age of the patients was 61.8 years. Age was similar in patients with microbleeds (61.6 years) and patients without microbleeds (62.2 years, $\mathrm{p}=0.936$ ).

Microbleeds were found in 23 patients (62\%) and $\mathrm{CH}$ in 26 patients $(70 \%)$. Both microbleeds and $\mathrm{CH}$ were found in 15 patients (41\%). Although the proportion of patients with microbleeds who had poor outcome (83\%) was larger than the proportion of patients without microbleeds who had poor outcome (64\%), this difference was not significant $(\mathrm{p}=$ 0.19). Similarly, although the proportion of patients with $\mathrm{CH}$ who had poor outcome $(85 \%)$ was larger
Table 1 Patients' clinical and demographic data $(\mathbf{N}=$ 37)

\begin{tabular}{lcc}
\hline & Number (\%) & Mean \pm SD \\
\hline Age (year) & $17-86$ & $61.8 \pm 22.1$ \\
Sex, male/female & $29 / 8$ & \\
GCS score before surgery & & $7.65 \pm 3.20$ \\
$3-8$ & $25(68 \%)$ & \\
$9-13$ & $10(27 \%)$ & \\
$14-15$ & $2(5 \%)$ & \\
Time of MR imaging from injury (day) & & $7.9 \pm 3.8$ \\
$0-1$ & $7(19 \%)$ & \\
$2-4$ & $4(11 \%)$ & \\
$5-9$ & $8(22 \%)$ & \\
$10-14$ & $18(49 \%)$ & \\
Operation side, right:left:both & $18: 16: 3$ & \\
\hline
\end{tabular}

GCS: Glasgow Coma Scale, MR: magnetic resonance, SD: standard deviation.

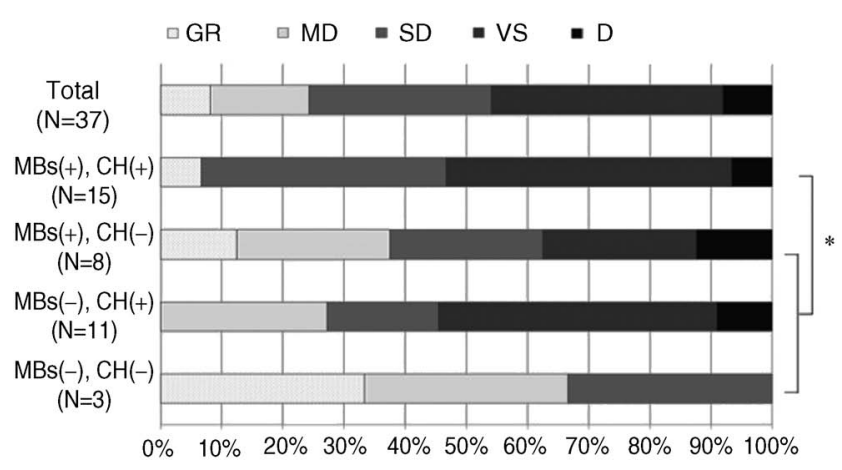

Fig. 2 Outcome at discharge in patients with and without microbleeds (MBs) and contusional hemorrhage (CH). Poor outcome at discharge was more common in patients with both $\mathrm{MBs}$ and $\mathrm{CH}$ than in patients with neither MBs nor CH $\left({ }^{*} p=0.04\right)$. D: $\operatorname{dead,~GR:~} \operatorname{good}$ recovery, MD: moderately disabled, SD: severely disabled, VS: vegetative state.

than the proportion of patients without $\mathrm{CH}$ who had poor outcome $(55 \%)$, this difference was not significant $(p=0.06)$. Of the 15 patients with both microbleeds and $\mathrm{CH}, 14$ (93\%) had poor outcome at discharge. This is a significantly higher proportion than that observed in patients who had either microbleeds or $\mathrm{CH}$, but not both $(64 \%, \mathrm{p}=0.04$, Fig. 2). Of 12 patients aged under 60 years, eight had microbleeds. Six of these $(75 \%)$ had poor outcome at discharge, whereas none of the four patients without microbleeds had poor outcome $(0 \%, p=0.03$, Fig. 3).

Microbleeds were located in lobar white matter or cerebellum only $(\mathrm{n}=12)$, in corpus callosum $(\mathrm{n}=$ 8), or in the brainstem ( $=5$ ) (Fig. 4). Two patients had microbleeds in both the corpus callosum and the brainstem. The location of the microbleed was not 


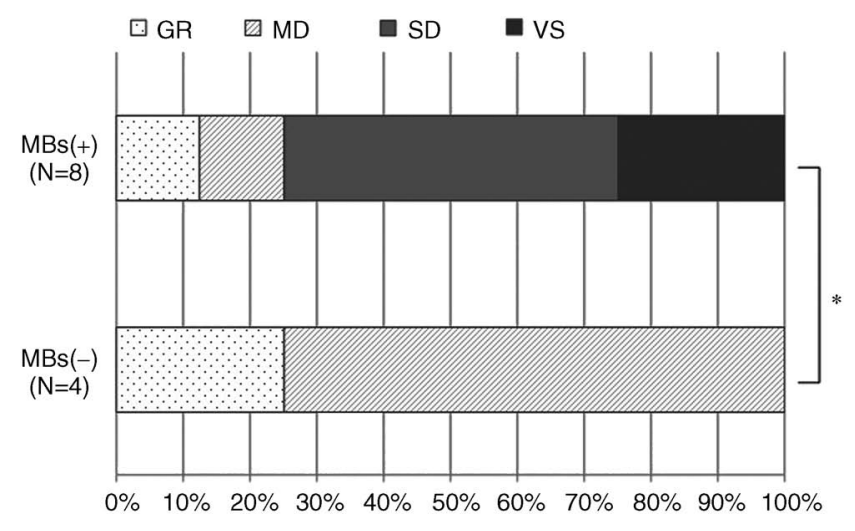

Fig. 3 Outcome at discharge in patients under 60 years old with and without microbleeds (MBs). Poor outcome at discharge was more common in patients with MBs than in patients without $\mathrm{MBs}\left({ }^{*} \mathrm{p}=\mathbf{0 . 0 3}\right)$. D: $\operatorname{dead}, \mathrm{GR}$ : good recovery, MD: moderately disabled, SD: severely disabled, VS: vegetative state.
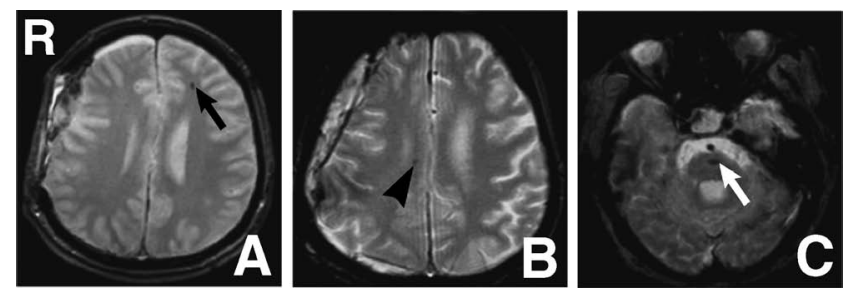

Fig. $4 \mathrm{~T}_{2}{ }^{*}$-weighted magnetic resonance images showing microbleeds in the lobar white matter (A: arrow), corpus callosum (B: arrowhead), and brainstem (C: arrow).

Table 2 Relationships between the location of microbleeds, contusional hemorrhage, and outcome

\begin{tabular}{lccc}
\hline & \multicolumn{3}{c}{ Location of microbleeds } \\
\cline { 2 - 4 } & $\begin{array}{c}\text { Lobar white } \\
\text { matter or } \\
\text { cerebellum }\end{array}$ & $\begin{array}{c}\text { Corpus } \\
\text { callosum }\end{array}$ & Brainstem \\
\hline Total & 12 & 8 & 5 \\
$\begin{array}{l}\text { Contusional hemorrhage } \\
\text { Outcome }\end{array} \quad 7(58 \%)$ & $6(75 \%)$ & $3(60 \%)$ \\
$\quad$ good & $1(8 \%)$ & $2(25 \%)$ & $2(40 \%)$ \\
poor & $11(92 \%)$ & $6(75 \%)$ & $3(60 \%)$ \\
\hline
\end{tabular}

related to the outcome at discharge (Table 2).

Illustrative Case 1, patient with microbleeds and CH: An 18-year-old man was admitted to our hospital after a traffic accident. On admission, Glasgow Coma Scale score was 6 with anisocoria, and head computed tomography (CT) revealed an acute SDH (Fig. 5A, B). Decompressive craniectomy was
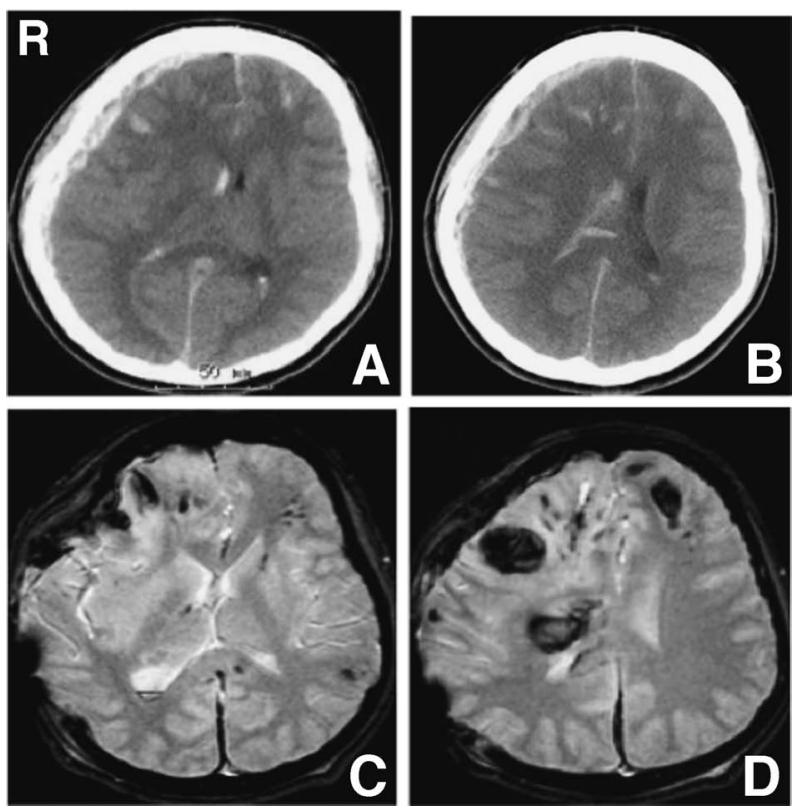

Fig. 5 Illustrative Case 1, an 18-year-old man who was admitted to hospital after a traffic accident. A, B: Computed tomography scans on admission showing right acute subdural hematoma. $\mathrm{C}, \mathrm{D}: \mathrm{T}_{2}{ }^{*}$-weighted magnetic resonance images obtained 8 days after admission showing microbleeds in the corpus callosum and lobar white matter, and contusional hemorrhage on both sides of the frontal lobe.
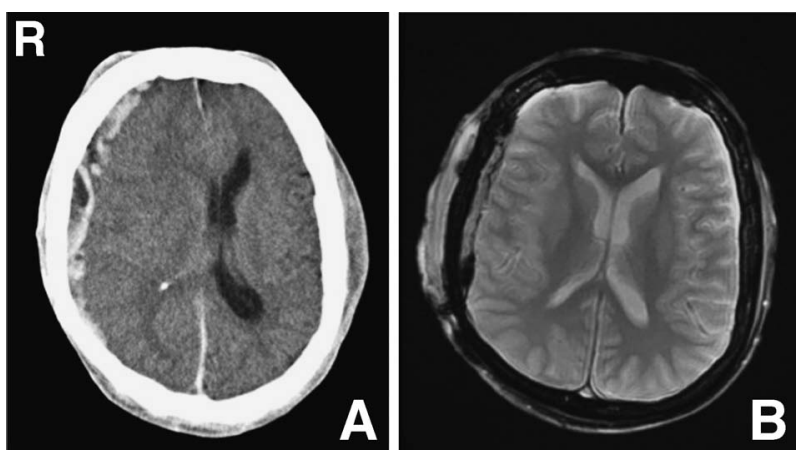

Fig. 6 Illustrative Case 2, a 50-year-old man who was admitted to hospital after a fall. A: Computed tomography scan on admission showing right acute subdural hematoma. B: $\mathrm{T}_{2}{ }^{*}$-weighted magnetic resonance image obtained 8 days after admission showing neither microbleeds nor contusional hemorrhage.

urgently performed. MR imaging performed 8 days after surgery revealed microbleeds in the corpus callosum and lobar white matter, and $\mathrm{CH}$ in both sides of the frontal lobe (Fig. 5C, D). The patient was moved from our hospital in a vegetative state at 95 days after surgery. 
Illustrative Case 2, patient without microbleeds and CH: A 50-year-old man was admitted to our hospital after a fall. On admission, Glasgow Coma Scale score was 6 with anisocoria, and head CT revealed an acute SDH (Fig. 6A). Decompressive craniectomy was urgently performed. MR imaging performed 8 days after surgery revealed neither microbleeds nor $\mathrm{CH}$ (Fig. 6B). The patient was discharged with good recovery at 10 days after surgery.

\section{Discussion}

This study showed that concurrent occurrence of microbleeds and $\mathrm{CH}$ was related to increased likelihood of poor outcome in patients with acute SDH. In addition, patients with only microbleeds or only $\mathrm{CH}$ tended to be more likely to have poor outcome than patients with neither microbleeds nor CH. Age, Glasgow Coma Scale score, and pupillary reactivity are known prognostic indicators in patients with acute SDH. ${ }^{4,6)}$ In some studies, SDH volume and midline brain shift have also been related to poor outcome. ${ }^{4,9,14)}$ This study is the first to report the effect of microbleeds on prognosis in patients with acute SDH. Our results support a previous study that reported a relationship between microbleeds and prognosis in patients with moderate and severe traumatic brain injury and diffuse axonal injury. ${ }^{3,19)}$

The majority of SDH cases that require surgery are complicated by associated intracranial and extracranial injuries, of which contusions and intracerebral hematomas are the most frequent.4) In this study, contusions and intracerebral hematomas were considered $\mathrm{CH}$, whereas diffuse axonal injuries and microvascular injuries were considered microbleeds. In autopsy after head injury, axonal shear-strain deformations are induced by sudden traumatic acceleration or rotational forces on the brain. The resulting injuries tend to be diffuse and accompanied by petechial hemorrhages ${ }^{1,2)}$ that can be detected as microbleeds on $\mathrm{T}_{2}{ }^{*}$-weighted MR images. Brain parenchymal injury markedly increases the mortality associated with SDH: SDH associated with brain parenchymal injury had a mortality of $53 \%$, compared to $9 \%$ for SDH without such injury. ${ }^{8)}$ We believe that, in the present study, patients with both microbleeds and $\mathrm{CH}$ frequently had poor outcome because they had complications that were caused by contusions, intracerebral hematomas, and diffuse axonal injuries.

The overall prevalence of microbleeds is high, and is reported to increase with age from $18 \%$ in persons aged $60-69$ years to $38 \%$ in those over 80 years. ${ }^{17)} \mathrm{We}$ found that, even in patients under 60 years of age, poor outcome at discharge was more common in patients with microbleeds than in patients without microbleeds. Therefore, regardless of age, poor outcome at discharge tended to be more common in patients with microbleeds than in patients without microbleeds.

It has been reported that there is a correlation between poor outcome and lesion location in the brainstem or corpus callosum in patients with diffuse axonal injury. ${ }^{3,11)}$ In this study, there were no differences in outcome according to the location of microbleeds. However, the small number of patients with microbleeds in the corpus callosum and the brainstem limits this comparison, and more patients are needed to address this point.

The conclusions of this study may be limited by selection bias, because patients who were conservatively treated or did not receive MR imaging within 2 weeks of head injury onset were not considered. These patients were considered to be not severe enough to perform surgery or MR imaging. Thus, our study may have included many patients with poor outcome. Furthermore, the timing of MR imaging was not constant across subjects. Microbleeds are perivascular collections of hemosiderin deposits. ${ }^{5)} \mathrm{T}_{2}{ }^{*}$-weighted MR imaging detects the paramagnetic effects of deoxyhemoglobin and methemoglobin, ${ }^{10)}$ but cannot detect the diamagnetic effects of oxyhemoglobin. Oxyhemoglobin changes to deoxyhemoglobin during the hyperacute phase. In this study, MR imaging was performed before surgery, i.e., during the hyperacute phase, in seven patients. It is possible that microbleeds increased with progress after surgery; however, we cannot evaluate this because MR imaging was performed on only one occasion. Considering the appearance of deoxyhemoglobin and methemoglobin, the appropriate time for MR imaging is 3-7 days after injury. In our study, eight patients underwent MR imaging during this period. Four of five patients $(80 \%)$ with microbleeds had poor outcome, whereas one of three patients (33\%) without microbleeds had poor outcome. Susceptibility-weighted MR imaging is a highly sensitive way of identifying microbleeds and diffusion tensor imaging is a sensitive way of evaluating white matter lesions. ${ }^{15)}$ Examination of prognosis using these methods is required in patients with acute SDH, as these methods can detect minute brain damages which cannot be detected by $\mathrm{T}_{2}{ }^{*}$-weighted MR imaging. To resolve these limitations, prospective studies should be performed to examine outcomes in SDH patients with and without microbleeds.

In conclusion, the results of this study demonstrate that poor outcome at discharge is more common in SDH patients with both microbleeds and $\mathrm{CH}$ 
than in SDH patients with neither microbleeds nor $\mathrm{CH}$, regardless of patient age and microbleed location. Therefore, the presence of microbleeds and $\mathrm{CH}$ on MR images may indicate poor prognosis in patients with acute SDH.

\section{Conflicts of Interest Disclosure}

The authors have no personal financial or institutional interest in any of the drugs, materials, or devices in the article. All authors who are members of The Japan Neurosurgical Society (JNS) have registered online Self-reported COI Disclosure Statement Forms through the website for JNS members.

\section{References}

1) Adams JH: Pathology of nonmissile head injury. Neuroimaging Clin North Am 1: 397-410, 1991

2) Adams JH, Greham DI, Murray LS, Scott G: Diffuse axonal injury due to nonmissile head injury in humans: analysis of 45 cases. Ann Neurol 12: 557-563, 1982

3) Alfonso L, Ana R, Angel PN, Federico B, Rafael A, Pedro AG, Ariel K, Ramoro DL: The MR imaging in assessing prognosis after severe and moderate head injury. Acta Neurochir (Wien) 151: 341-356, 2009

4) Bullock MR, Chesnut R, Ghajar J, Gordon D, Hartl R, Newell DW, Servadei F, Walters BC, Wilberger JE: Surgical management of subdural hematomas. Neurosurgery 58: S16-24, 2006

5) Cordonnier C: Brain microbleeds. Pract Neurol 10: 94-100, 2010

6) Dent DL, Croce MA, Menke PG, Young BH, Hinson MS, Kudsk KA, Minard G, Pritchard FE, Rodertson JT, Febian TC: Prognostic factors after acute subdural hematoma. J Trauma 39: 36-43, 1995

7) Gerber DJ, Weintraub AH, Cusick CP, Ricci PE, Whiteneck GG: Magnetic resonance imaging of traumatic brain injury: relationship of $\mathrm{T} 2 * \mathrm{SE}$ and T2GE to clinical severity and outcome. Brain Inj 18: 1083-1097, 2004

8) Hlatky R, Valadka AB, Goodman JC, Robertson CS: Evolution of brain tissue injury after evacuation of acute traumatic subdural hematomas. Neurosurgery 55: 1318-1324, 2004

9) Howard MA 3rd, Gross AS, Dacey RJ Jr, Winn HR: Acute subdural hematomas: an age-dependent clinical entity. J Neurosurg 71: 858-863, 1989

10) Kidwell CS, Chalela JA, Saver JL, Starkman S, Hill MD, Demchuk AM, Butman JA, Patronas N, Alger JR, Latour LL, Luby ML, Baird AE, Leary MC, Tremwel M, Ovbiagele B, Fredieu A, Suzuki S, Villablanca JP, Davis S, Dunn B, Todd JW, Ezzeddine MA, Hay- more J, Lynch JK, Davis L, Warach S: Comparison of MRI and CT for detection of acute intracerebral hemorrhage. JAMA 292: 1823-1830, 2004

11) Matsukawa $H$, Shinoda $M$, Fujii $M$, Takahashi $O$, Yamamoto D, Murakata A: Genu of corpus callosum as a prognostic factor in diffuse axonal injury. J Neurosurg 115: 1019-1024, 2011

12) Seelig JM, Becker DP, Miller JD, Greenberg RP, Ward JD, Choi SC: Traumatic acute subdural hematoma: major mortality reduction in comatose patients treated within four hours. N Engl J Med 304: 1511-1518, 1981

13) Servadei F, Nasi MT, Cremonini AM, Giuliani G, Cenni P, Nanni A: Importance of a reliable admission Glasgow Coma Scale score for determining the need for evacuation of posttraumatic subdural hematomas: a prospective study of 65 patients. J Trauma 44: 868-873, 1998

14) Sevadei F, Nasi MT, Giuliani G, Cremonini AM, Cenni P, Zappi D, Taylor GS: CT prognostic factors in acute subdural haematomas: the value of the 'worst' CT scan. Br J Neurosurg 14: 110-116, 2000

15) Sharp DJ, Ham TE: Investigating white matter injury after mild traumatic brain injury. Curr Opin Neurol 24: 558-563, 2011

16) Tong KA, Ashwal S, Holshouser BA, Nickerson JP, Wall CJ, Shutter LA, Osterdock RJ, Haacke EM, Kido D: Diffuse axonal injury in children: clinical correlation with hemorrhagic lesions. Ann Neurol 56: 36-50, 2004

17) Vernooij MW, van der Lugt A, Ikram MA, Wielopolski PA, Niessen WJ, Hofman A, Krestim GP, Breteler MMB: Prevalence and risk factors of cerebral microbleeds. Neurology 70: 1208-1214, 2008

18) Wilberger JE Jr, Harris M, Diamond DL: Acute subdural hematoma: morbidity, mortality, and operative timing. J Neurosurg 74: 212-218, 1991

19) Yanagawa $Y$, Sakamoto $T$, Takasu A, Okada Y: Relationship between maximum intracranial pressure and traumatic lesions detected by $\mathrm{T} 2{ }^{*}$-weighted imaging in diffuse axonal injury. J Trauma 66: 162-165, 2009

20) Yanagawa $Y$, Tsushima $Y$, Tokumaru A, Un-no $Y$, Sakamoto T, Okada Y, Nawashiro H, Shima K: A quantitative analysis of head injury using $\mathrm{T} 2{ }^{*}$ weighted gradient-echo imaging. J Trauma 49: 272-277, 2000

Address reprint requests to: Tadashi Yamaguchi, MD, Department of Neurosurgery, Kawasaki Medical School, 577 Matsushima, Kurashiki, Okayama 701-0192, Japan.

e-mail: tadashi117117@hotmail.co.jp 